\title{
MODULI OF RING DOMAINS OBTAINED BY A CONFORMAL WELDING
}

\author{
KinJiRo Nishikawa AND Fumio Maitani
}

\begin{abstract}
We are concerned with ring domains which are conformally welded along a pair of opposite sides of a square. Oikawa studied moduli of these ring domains and left some problems. We shall answer one of these open problems.
\end{abstract}

\section{Introduction}

Welding of polygons and the type of Riemann surfaces were considered by Nevanlinna, Oikawa and others (cf. [3], [4]). We are concerned with the relation of weldings and the moduli of Riemann surfaces. Oikawa studied this subject and got some results which he didn't publish (cf. [5]). We follow him. A square in the complex plane can be conformally welded into various ring domains by a specific kind of identification of a pair of opposite sides. We consider the range of these moduli. Oikawa gave an estimate for the range of these moduli and asked whether it is the best possible or not. We shall show a certain identification which give conformally welded ring domains with arbitrary small moduli. In addition, we shall show that the moduli of ring domains conformally welded by an unnatural identification never meet to a neighborhood of the module of ring domains conformally welded by the natural identification.

Consider the square $Q=\{x+i y: 0<x<1,0<y<1\}$ in the complex plane, and put

$$
L_{+}=\{x+i y: 0<x<1, y=1\}, \quad L_{-}=\{x+i y: 0<x<1, y=0\} .
$$

Let $\phi_{0}(x+i)=x(0<x<1)$ and $\phi: L_{+} \rightarrow L_{-}$be a homeomorphism such that $\phi^{\circ} \phi_{0}^{-1}(x)$ is strictly increasing. Let $G$ be a ring domain and $C$ be a Jordan curve in $G$ joining two boundary components of $G$. Let $f$ be a continuous mapping from $Q \cup L_{+} \cup L_{-}$onto $G$. We say the triple $(G, C, f)$ is a conformal welding obtained by $\phi$ if $f$ is conformal in $Q, f \circ \phi=f$ on $L_{+}$, and $f\left(L_{+}\right)=f\left(L_{-}\right)=C$. And we call $\phi$ a welding function. We say a conformal welding by $\phi$ is unique, if, for any two conformal weldings $\left(G_{\imath}, C_{\imath}, f_{\imath}\right)_{i=1,2}$ obtained by $\phi, f_{2} \circ f_{1}^{-1}$ is a con-

Received April 30, 1996 ; revised September 12, 1997. 
formal mapping from $G_{1}$ to $G_{2}$.

If a ring domain $G$ is conformally equivalent to an annulus $\left\{z: R_{1}<|z|<\right.$ $\left.R_{2}\right\}$, then the quantity $M(G)=\log \left(R_{2} / R_{1}\right)$ is called the modulus of $G$. The $M(G)$ is represented by means of extremal length. Let $\Lambda(G)$ be the set of Borel measurable conformal densities on $G$ and $\Gamma(G)$ be the set of rectifiable closed curves in $G$ separating the boundary components of $G$. For $\rho \in \Lambda(G)$, set

$$
\begin{gathered}
A(\rho, G)=\iint_{G} \rho(z)^{2} d x d y,(z=x+i y) \\
L(\rho, \Gamma(G))=\inf _{\gamma \in \Gamma(G)} \int_{\gamma} \rho(z)|d z| .
\end{gathered}
$$

Then

$$
\lambda(\Gamma(G))=\sup _{\rho \in \Lambda(G)} \frac{L(\rho, \Gamma(G))^{2}}{A(\rho, G)}
$$

is called the extremal length of $\Gamma(G)$ on $G$. We know $M(G)=2 \pi / \lambda(\Gamma(G))$.

For a welding function $\phi$, put

$$
M_{\phi}=\{M(G):(G, C, f) \text { is a conformal welding obtained by } \phi\} .
$$

If a conformal welding by $\phi$ is unique, then $M_{\phi}$ consists of a single point. On the other hand, if the 2-dimensional measure of $C$ is positive, then a conformal welding by $\phi$ is not unique. In fact, by using a variational formula (cf. Gardinar [1]), we know that $M_{\phi}$ contains an interval. Oikawa remaked that there is a welding function $\phi$ which has two conformal weldings $(G, C, f)$ and $\left(G^{\prime}\right.$, $\left.C^{\prime}, f^{\prime}\right)$ such that the area $|C|$ of $C$ is positive but the area $\left|C^{\prime}\right|$ of $C^{\prime}$ is zero. So the following Oikawa's problem is interesting for us.

Is there a welding function $\phi$ such that a conformal welding by $\phi$ is not unique, but $M_{\phi}$ consists of a single point?

Now Oikawa [5] proved the following three theorems.

THEOREM A. $M_{\phi}=\{2 \pi\}$ if and only if $\phi=\phi_{0}$.

THEOREM B. Let $\Phi$ be the set of welding functions $\phi$, then

$$
\bigcup_{\phi \in \Phi} M_{\phi}=(0,2 \pi] \text {. }
$$

For the sake of simplicity, we will often denote $\phi \circ \phi_{0}^{-1}(x)$ by $\phi(x)$.

THEOREM C.

$$
M_{\phi} \subset\left[2 \pi \int_{0}^{1} \frac{\min \left(\phi^{\prime}(x), 1\right)}{1+(\phi(x)-x)^{2}} d x, 2 \pi\right] .
$$

Further Oikawa [5] presented the following problem.

Find a welding function $\phi$ satisfying 


$$
M_{\phi} \supset\left(2 \pi \int_{0}^{1} \frac{\min \left(\phi^{\prime}(x), 1\right)}{1+(\phi(x)-x)^{2}} d x, 2 \pi\right) .
$$

In this paper, we shall show the following theorems, which answer this problem.

THEOREM 1. There are a welding function $\phi$ and $\varepsilon>0$ such that

$$
M_{\phi} \supset(0, \varepsilon) .
$$

THEOREM 2. For any $\phi \neq \phi_{0}$, there is an $m<2 \pi$ such that

$$
M_{\phi} \subset(0, m] \text {. }
$$

\section{An example of a welding function which yields ring domains with arbitrary small moduli}

In this section we prove Theorem 1. Let $A$ be an annulus. Then, for any $\varepsilon>0$ we can construct a Jordan curve $K$ in $A$ and a quasiconformal (=q.c.) mapping $h$ from $A$ onto a ring domain $G_{1}$ such that

(1) $K$ joins two boundary components of $A$,

(2) $h$ is conformal on $A-K$ and $M\left(G_{1}\right)<\varepsilon$.

Take a closed rectilinear triangle $K_{1}=\triangle a_{1} a_{2} a_{3}$ such that $K_{1}-\left\{a_{2}, a_{3}\right\} \subset A$ and $a_{2}, a_{3}$ lie on the different boundary components of $A$. Choose a vertex $a_{1}$ and take a segment $\left[b_{1}, b_{2}\right]$ on the edge $\left[a_{2}, a_{3}\right]$ which contains the midpoint of $\left[a_{2}, a_{3}\right]$. Delete the open triangle $\triangle_{1}=\triangle a_{1} b_{1} b_{2}$ from $K_{1}$ and put $K_{2}=K_{1}-\triangle_{1}=$ $K_{2}^{1} \cup K_{2}^{2}$, where $K_{2}^{\jmath}(j=1,2)$ is the closed triangle $\triangle b_{j} a_{1} a_{j+1}$. Next take an open triangle $\triangle_{2}^{j}=\triangle b_{j} c_{j 1} c_{j 2}$ on $K_{2}^{\jmath}(j=1,2)$, where $c_{j 1}$ and $c_{j 2}$ lie on the edge $\left[a_{1}, a_{\jmath+1}\right]$ and the edge $\left[c_{j 1}, c_{j 2}\right]$ contains the midpoint of the edge $\left[a_{1}, a_{\jmath_{+1}}\right]$. Set $K_{3}=$

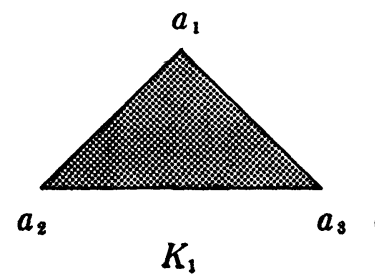

$K_{1}$

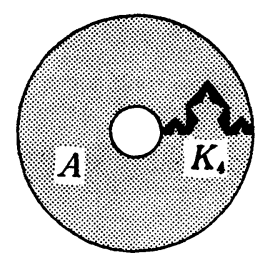

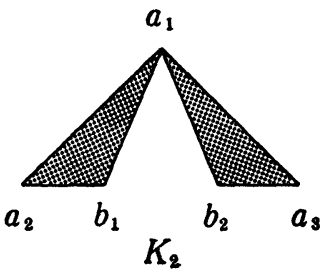

$K_{2}$
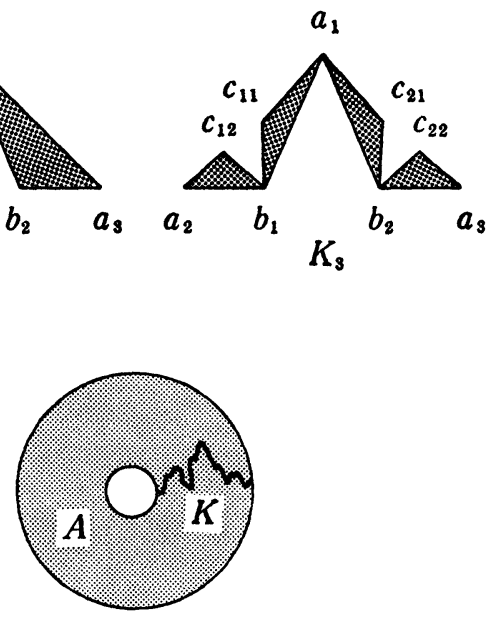
$\bigcup_{j=1,2}\left(K_{2}^{J}-\triangle_{2}^{J}\right)$. We repeat these processes to get a decreasing sequence of connected compact sets $\left\{K_{n}\right\}$. Then $K=\cap K_{n}$ becomes a Jordan curve, which is a kind of Koch curve. To attain our result, we must choose the deleted triangles in a certain manner, which is descrived after Lemma 1.

Lemma 1. Let $A$ be an annulus and $K_{n}$ be constructed as above. For any $\varepsilon>0$, there exists a q.c. mapping $h$ on $A$ which is conformal on $A-K_{n}$ and satisfies $M(h(A))<\varepsilon$.

Proof. Set $l=2^{n-1}$. The interior of $K_{n}$ consists of disjoint $l$ open triangles $\left\{O_{n}^{m}\right\}_{m=1,2, \ldots, l}$. Take a set of closed triangles $\left\{T_{m}\right\}(m=1,2, \ldots, l)$ such that each $T_{m}$ is contained in $O_{n}^{m}$. Let $K_{n}^{m}$ be the closure of $O_{n}^{m}$. Then $K_{n}=\bigcup_{m=1}^{l} K_{n}^{m}$. Let $g$ be a conformal mapping from $(l+2)$-connected domain $A-\cup T_{m}$ to an annulus $\Omega=\Omega\left(\left\{T_{m}\right\}\right)$ such that $\Omega-g\left(A-\cup T_{m}\right)$ consists of concentric circular slits. The boundary components of $\Omega$ are denoted by $C_{0}, C_{1}, \ldots, C_{l_{+1}}$, and the radius of the circle on which $C_{\imath}$ lies are denoted by $R_{\imath}$, where $R_{0}<R_{1} \leqq \cdots \leqq$ $R_{l}<R_{l+1}$.

Suppose that there exists a positive number $\alpha$ such that

$$
\log \frac{R_{l+1}}{R_{0}} \geqq \alpha
$$

for $\Omega\left(\left\{T_{m}\right\}\right)$ made by arbitrary choosen $\left\{T_{m}\right\}$. We can choose $p$ so that

$$
\log \frac{R_{p+1}}{R_{p}} \geqq \frac{\alpha}{l+1} .
$$

Let $\gamma_{i}(i=0,1, \ldots, p-1, p+1, \ldots l)$ be a set of Jordan curves such that each $\gamma_{2}$ joins $C_{\imath}$ to $C_{\imath+1}, \gamma_{i}(i \leqq p-1)$ is contained in $\left\{z: R_{0}<|z|<R_{p}\right\}$ and $\gamma_{i}(i \geqq p+1)$ is contained in $\left\{z: R_{p+1}<|z|<R_{l_{+1}}\right\}$. We can get a ring domain $\tilde{A}=\tilde{A}\left(\left\{T_{m}\right\},\left\{\gamma_{i}\right\}\right)$ $=g^{-1}\left(\Omega-\cup_{\imath \neq p} \gamma_{\imath}\right)$. It is proved in [2] that if the spherical diameters of the boundary components of $\tilde{A}$ are larger than $\tilde{d}$ and if their spherical distance is smaller than $d$, then

$$
M(\tilde{A}) \leqq \pi^{2}\left(\log \tan \frac{\tilde{d}}{2}-\log \tan \frac{d}{2}\right)^{-1} .
$$

Hence

$$
M(\tilde{A})=M\left(\Omega-\bigcup_{\imath \neq p} \gamma_{\imath}\right)
$$

tends to zero if $\operatorname{dist}\left(T_{p}, T_{p+1}\right)$ tends to zero. On the other hand,

$$
\log \frac{R_{p_{+1}}}{R_{p}}=M\left(\left\{z: R_{p}<|z|<R_{p+1}\right\}\right) \leqq M\left(\Omega-\bigcup_{i \neq p} \gamma_{\imath}\right) .
$$

This is a contradiction. Hence there exists $\left\{T_{m}\right\}$ such that $\log \left(R_{l+1} / R_{0}\right)$ with respect to $\Omega\left(\left\{T_{m}\right\}\right)$ is arbitrarily small.

Next take a set of simply connected domains $\left\{B_{m}\right\}$ such that each $B_{m}$ con- 
tains $T_{m}$, each $\partial B_{m}$ is real analytic and $B_{m} \cup \partial B_{m}$ is contained in the interior of $K_{n}^{m}$. Let $g_{1, m}$ and $g_{2, m}$ be conformal mappings from $B_{m}$ and $g\left(B_{m}-T_{m}\right) \cup$ $C_{m}$ to the unit disk $D$ respectively. Then $f_{0}=g_{2, m} \circ g \circ g_{1, m}^{-1}$ has a conformal extension to a neighborhood of $\partial D$. Hence

$$
\frac{\partial}{\partial \theta} \log f_{0}\left(e^{i \theta}\right)=i \frac{\partial}{\partial \theta} \arg f_{0}\left(e^{i \theta}\right) \neq 0 .
$$

Therefore $\eta(\theta)=\arg f_{0}\left(e^{i \theta}\right)$ is real analytic and $\eta^{\prime}(\theta)$ satisfies $\eta^{\prime}(\theta) \geqq \varepsilon>0$. We define a mapping $F_{0}: D \rightarrow D$ by $F_{0}\left(r e^{i \theta}\right)=r e^{i \eta(\theta)}$, where $x+i y=r e^{i \theta}$. Put $F=$ $(\log ) \circ F_{0} \circ(\exp )$ i.e., $F(x+i y)=x+i \eta(y)$. Therefore

$$
\left|\frac{F_{\bar{z}}}{F_{z}}\right|=\left|\frac{1-\eta^{\prime}(y)}{1+\eta^{\prime}(y)}\right| \leqq\left|\frac{1-\varepsilon}{1+\varepsilon}\right|<1 \text {. }
$$

Consider

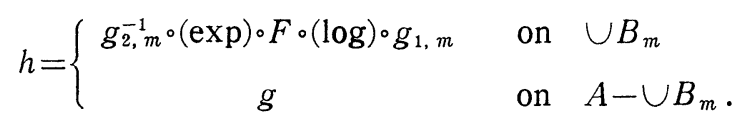

Then $h: A \rightarrow \Omega$ is a q.c. mapping which is conformal in $A-K_{n}$ and satisfies

$$
m(h(A))<\varepsilon .
$$

q.e.d.

We construct a Jordan curve $K$ and a q.c. mapping $h$ satisfying (1), (2). By Lemma 1 , there exists a q.c. mapping $h_{1}$ on $A$ which is conformal on $A-$ $K_{1}$ and satisfies

$$
M\left(h_{1}(A)\right)<\frac{M(A)}{2^{4}} .
$$

Let $\mu_{1}=\left(h_{1}\right)_{\bar{z}} /\left(h_{1}\right)_{z}$ be the Beltrami coefficient of $h_{1}$. Since

$$
\begin{gathered}
M\left(h_{1}(A)\right)=2 \pi \inf _{\rho \in \Lambda_{\left(h_{1}(A)\right)}} \frac{\iint_{h_{1}(A)} \rho(w)^{2} d u d v}{\left(\inf _{\gamma \in \Gamma\left(h_{1}(A)\right)} \int_{\gamma} \rho(w)|d w|\right)^{2}} \\
=2 \pi \inf _{\rho \in \Lambda_{\left(h_{1}(A)\right)}} \frac{\iint_{A} \rho\left(h_{1}(z)\right)^{2}\left|\left(h_{1}\right)_{z}(z)\right|^{2}\left|1-\mu_{1}(z)\right|^{2} d x d y}{\left(\inf _{\gamma \in \Gamma(A)} \int_{\gamma} \rho\left(h_{1}(z)\right)\left|\left(h_{1}\right)_{z}(z)\right|\left|d z+\mu_{1}(z) d \bar{z}\right|\right)^{2}},
\end{gathered}
$$

we can choose a conformal density $\rho_{1} \in \Lambda\left(h_{1}(A)\right)$ so that

$$
2 \pi \frac{A\left(\rho_{1}, h_{1}(A)\right)}{L\left(\rho_{1}, \Gamma\left(h_{1}(A)\right)\right)^{2}}<\frac{M(A)}{2^{4}} .
$$

In our above construction, by choosing the segment $\left[b_{1}, b_{2}\right]$ sufficiently short, we can take a compact set $K_{2}$ so that

$$
\iint_{K_{1}-K_{2}} \rho_{1}\left(h_{1}\right)^{2}\left|\left(h_{1}\right)_{z}\right|^{2}\left|\mu_{1}\right|^{2} d x d y \leqq \frac{1}{2} \iint_{A} \rho_{1}\left(h_{1}\right)^{2}\left|\left(h_{1}\right)_{z}\right|^{2}\left|1-\mu_{1}\right|^{2} d x d y .
$$

Again, by Lemma 1 , there exists a q.c. mapping $h_{2}$ on $A$ which is conformal on $A-K_{2}$ and satisfies 


$$
M\left(h_{2}(A)\right)<\frac{M(A)}{2^{5}} .
$$

Let $\mu_{2}=\left(\left(h_{2}\right)_{\bar{z}} /\left(h_{2}\right)_{z}\right)$ be the Beltrami coefficient of $h_{2}$. We can take a conformal density $\rho_{2} \in \Lambda\left(h_{2}(A)\right)$ such that

$$
2 \pi \frac{A\left(\rho_{2}, h_{2}(A)\right)}{L\left(\rho_{2}, \Gamma\left(h_{2}(A)\right)\right)^{2}}<\frac{M(A)}{2^{5}} .
$$

Now suppose that for $n \geqq 3,\left\{K_{l}\right\}_{l \leqq n-1},\left\{h_{l}\right\}_{l \leqq n-1},\left\{\mu_{l}\right\}_{l \leqq n-1},\left\{\rho_{l}\right\}_{l \leq n-1}$ satisfy the following :

$$
\begin{aligned}
& \iint_{K_{l-1}-K_{l}} \rho_{l-j}\left(h_{l-j}\right)^{2}\left|\left(h_{l-j}\right)_{z}\right|^{2}\left|\mu_{l-\jmath}\right|^{2} d x d y \\
& \leqq \frac{1}{2^{j}} \iint_{A} \rho_{l-j}\left(h_{l-\jmath}\right)^{2}\left|\left(h_{l-\jmath}\right)_{z}\right|^{2}\left|1-\mu_{l-j}\right|^{2} d x d y,
\end{aligned}
$$

for $1 \leqq j \leqq l-1$,

$$
M\left(h_{l}(A)\right)<\frac{M(A)}{2^{l+3}}, \quad \mu_{l}=\frac{\left(h_{l}\right)_{\bar{z}}}{\left(h_{l}\right)_{z}}, \quad 2 \pi \frac{A\left(\rho_{l}, h_{l}(A)\right)}{L\left(\rho_{l}, \Gamma\left(h_{l}(A)\right)\right)^{2}}<\frac{M(A)}{2^{l+3}} .
$$

Then we can take a compact set $K_{n}$ such that

$$
\begin{aligned}
& \iint_{K_{n-1}-K_{n}} \rho_{n-j}\left(h_{n-j}\right)^{2}\left|\left(h_{n-\jmath}\right)_{z}\right|^{2}\left|\mu_{n-\jmath}\right|^{2} d x d y \\
& \leqq \frac{1}{2^{\jmath}} \iint_{A} \rho_{n-j}\left(h_{n-j}\right)^{2}\left|\left(h_{n-\jmath}\right)_{z}\right|^{2}\left|1-\mu_{n-j}\right|^{2} d x d y,
\end{aligned}
$$

for $1 \leqq j \leqq n-1$. By Lemma 1 , we can get $h_{n}, \mu_{n}$ and $\rho_{n}$ such that

$$
M\left(h_{n}(A)\right)<\frac{M(A)}{2^{n+3}}, \quad \mu_{n}=\frac{\left(h_{n}\right)_{\bar{z}}}{\left(h_{n}\right)_{z}}, \quad 2 \pi \frac{A\left(\rho_{n}, h_{n}(A)\right)}{L\left(\rho_{n}, \Gamma\left(h_{n}(A)\right)\right)^{2}}<\frac{M(A)}{2^{n+3}} .
$$

Thus we can get a Jordan curve $K=\cap K_{n}$ which satisfies

$$
\iint_{K_{i}-K} \rho_{i}\left(h_{\imath}\right)^{2}\left|\left(h_{\imath}\right)_{z}\right|^{2}\left|\mu_{i}\right|^{2} d x d y \leqq \iint_{A} \rho_{i}\left(h_{\imath}\right)^{2}\left|\left(h_{\imath}\right)_{z}\right|^{2}\left|1-\mu_{i}\right|^{2} d x d y
$$

for every $i$. This $K$ joins two boundary components of $A$ and has positive 2-dimensional measure.

Let $f=f_{n}$ be a q.c. mapping on $\boldsymbol{C}$ such that

$$
\nu=\frac{f_{\bar{z}}}{f_{z}}=\left\{\begin{array}{lll}
\mu_{n} & \text { on } & K \\
0 & \text { on } & C-K .
\end{array}\right.
$$

Let

$$
\tilde{\rho}_{n}(w)=\rho_{n}\left(h_{n} \circ f^{-1}(w)\right)\left|\frac{\left(h_{n}\right)_{z}\left(f^{-1}(w)\right)}{f_{z}\left(f^{-1}(w)\right)}\right|
$$

We have 


$$
\begin{aligned}
M(f(A)) & =2 \pi \inf _{\rho \in \Lambda(f(A))} \frac{\iint_{A} \rho(f(z))^{2}\left|f_{z}(z)\right|^{2}\left(1-|\nu(z)|^{2}\right) d x d y}{\left(\inf _{\gamma \in \Gamma(A)} \int_{\gamma} \rho(f(z))\left|f_{z}(z)\right||d z+\nu(z) d \bar{z}|\right)^{2}} \\
& \leqq 2 \pi \frac{\iint_{A} \tilde{\rho}_{n}(f(z))^{2}\left|f_{z}(z)\right|^{2}\left(1-|\nu(z)|^{2}\right) d x d y}{\left(\inf _{\gamma \in \Gamma(A)} \int_{\gamma} \tilde{\rho}_{n}(f(z))\left|f_{z}(z)\right||d z+\nu(z) d \bar{z}|\right)^{2}} \\
& =2 \pi \frac{\iint_{A} \rho_{n}\left(h_{n}(z)\right)^{2}\left|\left(h_{n}\right)_{z}(z)\right|^{2}\left(1-|\nu(z)|^{2}\right) d x d y}{\left(\inf _{\gamma \in \Gamma(A)} \int_{\gamma} \rho_{n}\left(h_{n}(z)\right)\left|\left(h_{n}\right)_{z}(z)\right||d z+\nu(z) d \bar{z}|\right)^{2}} .
\end{aligned}
$$

On one hand,

$$
\begin{aligned}
& \iint_{A} \rho_{n}\left(h_{n}(z)\right)^{2}\left|\left(h_{n}\right)_{z}(z)\right|^{2}\left(1-|\nu(z)|^{2}\right) d x d y \\
& =\iint_{A-\left(K_{n}-K\right)} \rho_{n}\left(h_{n}(z)\right)^{2}\left|\left(h_{n}\right)_{z}(z)\right|^{2}\left(1-\left|\mu_{n}(z)\right|^{2}\right) d x d y \\
& \quad+\iint_{\left(K_{n}-K\right)} \rho_{n}\left(h_{n}(z)\right)^{2}\left|\left(h_{n}\right)_{z}(z)\right|^{2} d x d y \\
& =\iint_{A} \rho_{n}\left(h_{n}(z)\right)^{2}\left|\left(h_{n}\right)_{z}(z)\right|^{2}\left(1-\left|\mu_{n}(z)\right|^{2}\right) d x d y \\
& \quad+\iint_{\left(K_{n}-K\right)} \rho_{n}\left(h_{n}(z)\right)^{2}\left|\left(h_{n}\right)_{z}(z)\right|^{2}\left|\mu_{n}(z)\right|^{2} d x d y \\
& \leqq \iint_{A} \rho_{n}\left(h_{n}(z)\right)^{2}\left|\left(h_{n}\right)_{z}(z)\right|^{2}\left(1-\left|\mu_{n}(z)\right|^{2}\right) d x d y \\
& \quad+\iint_{A} \rho_{n}\left(h_{n}(z)\right)^{2}\left|\left(h_{n}\right)_{z}(z)\right|^{2}\left(1-\left|\mu_{n}(z)\right|^{2}\right) d x d y \\
& =2 \iint_{A} \rho_{n}\left(h_{n}(z)\right)^{2}\left|\left(h_{n}\right)_{z}(z)\right|^{2}\left(1-\left|\mu_{n}(z)\right|^{2}\right) d x d y .
\end{aligned}
$$

On the other hand,

$$
\begin{aligned}
& \int_{\gamma} \rho_{n}\left(h_{n}(z)\right)\left|\left(h_{n}\right)_{z}(z)\right||d z+\nu(z) d \bar{z}| \\
& =\int_{r \cap\left(C-\left(K_{n}-K\right)\right)} \rho_{n}\left(h_{n}(z)\right)\left|\left(h_{n}\right)_{z}(z)\right|\left|d z+\mu_{n}(z) d \bar{z}\right| \\
& \quad+\int_{r \cap\left(K_{n}-K\right)} \rho_{n}\left(h_{n}(z)\right)\left|\left(h_{n}\right)_{z}(z)\right||d z| \\
& \geqq \int_{r \cap\left(C-\left(K_{n}-K\right)\right)} \rho_{n}\left(h_{n}(z)\right)\left|\left(h_{n}\right)_{z}(z)\right|\left|d z+\mu_{n}(z) d \bar{z}\right| \\
& \quad+\frac{1}{2} \int_{r \cap\left(K_{n}-K\right)} \rho_{n}\left(h_{n}(z)\right)\left|\left(h_{n}\right)_{z}(z)\right|\left|d z+\mu_{n}(z) d \bar{z}\right|
\end{aligned}
$$


Therefore

$$
\begin{aligned}
M(f(A)) & \leqq 2 \pi \frac{2 \iint_{A} \rho_{n}\left(h_{n}(z)\right)^{2}\left|\left(h_{n}\right)_{z}(z)\right|^{2}\left(1-\left|\mu_{n}(z)\right|^{2}\right) d x d y}{\left(\inf _{\gamma \in \Gamma(A)}(1 / 2) \int_{\gamma} \rho_{n}\left(h_{n}(z)\right)\left|\left(h_{n}\right)_{z}(z)\right|\left|d z+\mu_{n}(z) d \bar{z}\right|\right)^{2}} \\
& =16 \pi \frac{A\left(\rho_{n}, \frac{\left.h_{n}(A)\right)}{L\left(\rho_{n}, \Gamma\left(h_{n}(A)\right)\right)^{2}}<\frac{M(A)}{2^{n}}\right.}{}
\end{aligned}
$$

which shows (2).

Proof of Theorem 1. Let $f_{t}$ be a q.c. mapping on $A$ which satisfies

$$
\frac{\left(f_{t}\right)_{\bar{z}}}{\left(f_{t}\right)_{z}}=t \nu(0 \leqq t \leqq 1) \text {. }
$$

By using the variational formula [1], we know

$$
\left\{M\left(f_{t}(A)\right) ; 0 \leqq t \leqq 1\right\} \supset\left[\frac{M(A)}{2^{n}}, M(A)\right] .
$$

Let $g_{3}$ be a conformal mapping from $A-K$ to a rectangle $Q_{0}$ whose horizontal sides correspond to $K, g_{4}$ be a q.c. mapping from $Q$ to $Q_{0}$ which preserve the vertical and horizontal sides. There exists a q.c. mapping $g_{5}$ on $A$ into $C$ such that $g_{5} \circ g_{3}^{-1} \circ g_{4}$ is conformal on $Q$. Let $k=\operatorname{esssup}\left|\left(g_{5}\right)_{\bar{z}} /\left(g_{5}\right)_{z}\right|<1$. Set $g=$ $g_{5} \circ g_{3}^{-1} \circ g_{4}, g_{5}(A)=G, g_{5}(K)=C$. Since $g$ has a continuous, injective extension $\tilde{g}$ to the boundary, we can define $\phi=\tilde{g}^{-1} \circ \tilde{g}: L_{+} \rightarrow L_{-}$. Then $(G, C, \tilde{g})$ becomes a conformal welding obtained from $Q$ by $\phi$. It follows that for sufficiently large $n$,

$$
M_{\phi} \supset\left[\frac{(1+k) M(A)}{(1-k) 2^{n}}, \frac{(1-k) M(A)}{(1+k)}\right] .
$$

Hence $M_{\phi}$ contains $(0,(1-k) M(A) /(1+k)]$.

Remark. Let a welding function $\phi$ satisfy Theorem 1 . Then by Theorem $\mathrm{C}$, we have

$$
\int_{0}^{1} \frac{\min \left(\phi^{\prime}(x), 1\right)}{1+(\phi(x)-x)^{2}} d x=0 .
$$

Hence $\phi^{\prime}(x)$ vanishes almost everywhere on $(0,1)$. Contrary, we don't know whether the vanishing of $\phi^{\prime}$ a.e. on $(0,1)$ implies that $M_{\phi} \supset(0, \varepsilon)$.

\section{Upper bound of the moduli for a welding function}

In this section we prove Theorem 2. Suppose that there exists a welding function $\phi$ such that the closure of $M_{\phi}$ contains $2 \pi$. There exist conformal 
weldings $\left(G_{n}, C_{n}, f_{n}\right)$ such that $r_{n}=M\left(G_{n}\right)$ increases monotonously to $2 \pi$. Here we may assume $G_{n}=\left\{z: 1<|z|<e^{r_{n}}\right\}$. Let $G=\left\{z: 1<|z|<e^{2 \pi}\right\}, f=e^{2 \pi} z$ and $C$ be an open interval $\left(1, e^{2 \pi}\right)$. Then $(G, C, f)$ is the conformal welding by the welding function $\phi_{0}(x+i)=x$. Each $f_{n}$ and $f$ have continuous extensions to the boundary of $Q$ by Carathéodory's theorem, which are denoted by the same symbols. Set

$$
w_{n}=\frac{1}{r_{n}} \log \left|f_{n}\right|, \quad w=\frac{1}{2 \pi} \log |f| .
$$

Since each $f_{n}$ is univalent and uniformly bounded, we can take a subsequence of $\left\{f_{n}\right\}$ which converges to a conformal mapping $f_{0}$ uniformly on each compact set in $Q$. We may assume that $f_{n}$ converges to $f_{0}$ uniformly on each compact set in $Q$. We can show the following.

LEMMA 2. $f_{0}=e^{\imath \alpha} f$, where $\alpha$ is a real constant.

Proof. Set $L_{a}=\{x+i a: 0<x<1\}$, where $0<a<1$. We have

$$
2 \pi \leqq \int_{L_{a}}\left|d \log f_{0}\right|=\int_{0}^{1} \frac{\left|f_{0}^{\prime}\right|}{\left|f_{0}\right|} d x .
$$

Hence

$$
4 \pi^{2} \leqq\left(\int_{0}^{1} \frac{\left|f_{0}^{\prime}\right|}{\left|f_{0}\right|} d x\right)^{2} \leqq \int_{0}^{1} d x \int_{0}^{1} \frac{\left|f_{0}^{\prime}\right|^{2}}{\left|f_{0}\right|^{2}} d x=\int_{0}^{1} \frac{\left|f_{0}^{\prime}\right|^{2}}{\left|f_{0}\right|^{2}} d x .
$$

Furthermore,

$$
\begin{aligned}
4 \pi^{2} & \leqq \int_{0}^{1} d y \int_{0}^{1} \frac{\left|f_{0}^{\prime}\right|^{2}}{\left|f_{0}\right|^{2}} d x=\iint_{Q} \frac{\left|f_{0}^{\prime}\right|^{2}}{\left|f_{0}\right|^{2}} d x d y \\
& =\iint_{f_{0}(Q)} \frac{d u d v}{|w|^{2}} \leqq \iint_{G} \frac{d u d v}{|w|^{2}}=4 \pi^{2} \quad(w=u+i v) .
\end{aligned}
$$

It follows that for almost all $a$ in $(0,1)$

$$
2 \pi=\int_{L_{a}}\left|d \log f_{0}\right|
$$

and $f_{0}\left(L_{a}\right)$ lies on a line through 0 . Since

$$
\iint_{f_{0}(Q)} \frac{d u d v}{|w|^{2}}=\iint_{G} \frac{d u d v}{|w|^{2}}
$$

$f_{0}\left(L_{+}\right)=f_{0}\left(L_{-}\right)$lies on a line through 0 with an argument $\alpha$ and

$$
f_{0}(Q)=\left\{z: 1<|z|<e^{2 \pi}\right\}-\left\{z: \arg z=\alpha, 1<|z|<e^{2 \pi}\right\} .
$$

Let $g_{6}$ denote the inverse mapping $f_{0}^{-1}$ from $f_{0}\left(L_{+}\right)$to $L_{+}$and $g_{7}$ from $f_{0}\left(L_{-}\right)$ to $L_{-}$. Set $\phi_{1}=g_{7} \circ g_{6}^{-1}$. Since $M\left(f_{0}(Q)\right)=2 \pi$, Theorem A implies $\phi_{1}=\phi_{0}$. Thus $f_{0} \circ f^{-1}$ is a conformal mapping on $G$. Hence $f_{0}=e^{\imath \alpha} f$. q.e.d. 
Lemma 3. The Dirichlet norm $\left\|d w_{n}\right\|_{Q}=\sqrt{\iint_{Q} d w_{n} \wedge d w_{n}}$ converges to $\|d w\|_{Q}$.

Proof. Set $L^{-}=\{i y: 0<y<1\}$ and $L^{+}=\{1+i y: 0<y<1\}$. Each conformal mapping $f_{n}$ on $Q$ has a conformal extension to a neighborhood of $L^{+}, L^{-}$. We denote the extension by the same symbol. For any positive number $\varepsilon$ there exists a rectangle $Q_{\varepsilon}=\left\{x+i y: 0<x<1,0<b_{\varepsilon} \leqq y \leqq b_{\varepsilon}^{\prime}<1\right\}$ such that

$$
\operatorname{Area}\left(f_{0}(Q)\right)-\operatorname{Area}\left(f_{0}\left(Q_{\varepsilon}\right)\right) \leqq \varepsilon \text {. }
$$

Since $\left\{f_{n}\right\}$ converges to $f_{0}$ uniformly on $Q_{\varepsilon}$, there exists an integer $N$ such that for all $n \geqq N$

Then

$$
\operatorname{Area}\left(f_{0}\left(Q_{\varepsilon}\right)\right)-\operatorname{Area}\left(f_{n}\left(Q_{\varepsilon}\right)\right) \leqq \varepsilon
$$

$$
\begin{aligned}
\operatorname{Area}\left(f_{n}\left(Q-Q_{\varepsilon}\right)\right) & =\operatorname{Area}\left(f_{n}(Q)\right)-\operatorname{Area}\left(f_{n}\left(Q_{\varepsilon}\right)\right) \\
& \leqq \operatorname{Area}\left(f_{0}(Q)\right)-\operatorname{Area}\left(f_{n}\left(Q_{\varepsilon}\right)\right) \\
& \leqq \operatorname{Area}\left(f_{0}(Q)\right)-\operatorname{Area}\left(f_{0}\left(Q_{\varepsilon}\right)\right)+\varepsilon \leqq 2 \varepsilon .
\end{aligned}
$$

Since $w_{n}=r_{n}^{-1} \log \left|f_{n}\right|, d w_{n}=r_{n}^{-1} \Re\left(d f_{n} / f_{n}\right)$. We have

$$
\begin{aligned}
\left\|d w_{n}\right\|_{Q}^{2} & =\left\|d w_{n}\right\|_{Q_{\varepsilon}}^{2}+\left\|d w_{n}\right\|_{Q-Q_{\varepsilon}}^{2} \leqq\left\|d w_{n}\right\|_{Q_{\varepsilon}}^{2}+\frac{1}{r_{n}^{2}}\left\|d f_{n}\right\|_{Q-Q_{\varepsilon}}^{2} \\
& =\left\|d w_{n}\right\|_{Q_{\varepsilon}}^{2}+\frac{1}{r_{n}^{2}} \operatorname{Area}\left(f_{n}\left(Q-Q_{\varepsilon}\right)\right) \leqq\left\|d w_{n}\right\|_{Q_{\varepsilon}}^{2}+2 \varepsilon .
\end{aligned}
$$

Therefore

$$
\begin{aligned}
& \|d w\|_{Q}^{2} \leqq \varlimsup_{n \rightarrow \infty}\left\|d w_{n}\right\|_{Q}^{2} \leqq \varlimsup_{n \rightarrow \infty}\left(\left\|d w_{n}\right\|_{Q_{\varepsilon}}^{2}+2 \varepsilon\right) \\
& =\|d w\|_{Q_{\varepsilon}}^{2}+2 \varepsilon \leqq\left\|d w_{n}\right\|_{Q}^{2}+2 \varepsilon .
\end{aligned}
$$

q.e.d.

Proof of Theorem 2. By the parallelogram law

$$
\begin{aligned}
\left\|d w_{n}-d w_{m}\right\|_{Q}^{2} & =2\left(\left\|d w_{n}\right\|_{Q}^{2}+\left\|d w_{m}\right\|_{Q}^{2}\right)-\left\|d w_{n}+d w_{m}\right\|_{Q}^{2} \\
& \leqq 2\left\{\left\|d w_{n}\right\|_{Q}^{2}+\left\|d w_{m}\right\|_{Q}^{2}-\|d w\|_{Q}^{2}\right\} \longrightarrow 0 .
\end{aligned}
$$

Hence $\left\{d w_{n}\right\}$ is a Cauchy sequence and converges to a differential $d w_{*}$. Since $\left\{w_{n}\right\}$ converges to $w$ uniformly on every compact set in $Q, d w_{*}=d w$.

Let $g$ be a conformal mapping from the unit disk $D$ to $Q$. Then $g$ has a continuous extension to the boundary of $D$ by Carathéodory's theorem, which is denoted by the same symbol. Set

$$
W_{n}=w_{n} \circ g-w_{n} \circ g(0), \quad W=w \circ g-w \circ g(0) .
$$

Then $\left\{d W_{n}\right\}$ converges to $d W$ with respect to Dirichlet norm on $D$. Since $W_{n}-W$ is harmonic on $D$, we can write it as 


$$
W_{n}-W=\sum_{k=1}^{\infty}\left(a_{k}(n) z^{k}+\overline{a_{k}(n)} \bar{z}^{k}\right) .
$$

We see that $\left\{W_{n}\right\}$ converges to $W$ on $\partial D$ in $L_{2}$-norm, because

$$
\left\|W_{n}-W\right\|_{\partial D}^{2}=4 \pi \sum_{k=1}^{\infty}\left|a_{k}(n)\right|^{2} \leqq 4 \pi \sum_{k=1}^{\infty} k\left|a_{k}(n)\right|^{2}=\left\|d W_{n}-d W\right\|_{D}^{2} .
$$

Now for every $n, W_{n} \circ g^{-1} \circ \phi=W_{n} \circ g^{-1}$ on $L_{+}$. The left hand side converges to $W \circ g^{-1} \circ \phi$ and the right hand side converges to $W \circ g^{-1}$ almost everywhere on $L_{+}$. Hence $\phi=\phi_{0}$ if the closure of $M_{\phi}$ contains $2 \pi$. This shows Theorem 2 .

Acknowledgement. We wish to express our gratitude to referee for the kind comments. We are also grateful to Professors Kunihiko Matsui and Hisashi Ishida for their valuable advice.

\section{REFERENCES}

[1] F. Gardiner, Schiffers interior variation and quasiconformal mappings, Duke Math. J., 42 (1975), pp. 373-380.

[2] O. Lehto and K.I. Virtanen, Quasiconformal Mapping in the Plane, SpringerVerlag, 1973.

[3] R. Nevanlinna, Polygonal representations of Riemann surface, Lectures on Functions of a Complex Variables, Univ. Michigan Press, 1955, pp. 65-70.

[4] K. OIKAWA, Welding of polygons and the type of Riemann surfaces, Kodai Math. Sem. Rep., 13 (1961), pp. 35-52.

[5] K. OiKawA, Moduli of Ring domains obtained by conformal sewing, Lecture memorial.

Hachiman High SchoOl

Ohumihachiman, Shiga 523

JAPAN

Kyoto Institute of Technology

SAKYOKU, KYOTO 606

JAPAN 